\title{
Asymptotic tunneling conductance in Luttinger liquids
}

\author{
F. Guinea \\ Instituto de Ciencia de Materiales. CSIC. \\ Cantoblanco. E-28049 Madrid. Spain. \\ G. Gómez-Santos \\ Departamento de Física de la Materia Condensada. \\ Universidad Autónoma. E-28049 Madrid. Spain. \\ M. Sassetti \\ Istituto di Fisica di Ingegneria, INFM. \\ Universitá di Genova. I-16146 Genova. Italy. \\ M. Ueda \\ Department of Physical Electronics, Hiroshima University, \\ Higashi-Hiroshima 724, Japan.
}

Conductance through weak constrictions in Luttinger liquids is shown to vanish with frequency $\omega$ as $c_{1} \omega^{2}+c_{2} \omega^{2 / g-2}$, where $g$ is a dimensionless parameter characterizing the Luttinger liquid phase, and $c_{1}$ and $c_{2}$ are nonuniversal constants. The first term arises from the 'Coulomb blockade' effect and dominates for $g<1 / 2$, whereas the second results from eliminating high-energy modes and dominates for $g>1 / 2$.

PACS numbers: 75.10.Jm, 75.10.Lp, 75.30.Ds.

Tunneling in Luttinger liquids has lately attracted a great deal of attention because this has been suggested to be the best way to characterize the Luttinger liquid phase and because a simple example of Luttinger liquids is nowadays accessible experimentally, the edge current in the fractional quantum Hall effect [1].

While a bulk Luttinger liquid is well understood [2], the tunneling problem has only recently been initiated by Kane and Fisher [3], who mapped the problem onto the Schmid model [4], which has extensively been studied in connection with Josephson junctions. They showed that the duality exhibited by this model is also applicable to the Luttinger problem. Then using a well-established renormalization-group (RG) approach [5.,6], they suggested that for the weak-barrier and repulsive case, the low-temperature conductivity of a junction between Luttinger liquids should vanish as $T^{2 / g-2}$, where $g$ is a dimensionless parameter which depends on the strength of the interactions (see below).

It is thus surprising that Mak and Egger [7], using the same model and mapping, suggested the $T^{2}$ law in the case of the weak-barrier and repulsive case on evidence from real-time Montecarlo simulations. In this case a finite cut-off is employed to extract the low temperature regime. Note that expansions around $g=1 / 2$, in which the cutoff is set to infinity give different results [8]. Other
Montecarlo work in imaginary-time, however, reported conflicting results [9].

In view of current experimental and theoretical efforts to understand the transport properties of Luttinger liquids, it seems urgent to clarify the situation. In this paper, we show that the asymptotic low frequency conductance, in general, obeys $c_{1} \omega^{2}+c_{2} \omega^{2 / g-2}$, and that depending on whether $g<1 / 2$ or $g>1 / 2$, one term dominates or the other.

Consider first the weak-barrier, attractive case. The Hamiltonian that describes a free bosonic field in a local periodic potential is given by [3, 8 ]

$$
\mathcal{H}=\frac{1}{2} \int d x\left(g \Pi(x)^{2}+g^{-1}\left(\partial_{x} \phi(x)\right)^{2}\right)+V \cos (\sqrt{4 \pi} \phi(0))
$$

where the harmonic field describes the dynamics of a perfect Luttinger liquid, and the periodic term describes the effect of the barrier. The dimensionless parameter $g$ characterizes the decay of the electronic Green functions and depends on the nature of the interactions: $g<1$ corresponds to repulsive interactions, and $g>1$ to attractive ones. As shown in Ref. [3], the bosonic degrees of freedom other than $\phi(0)$ can be integrated out, and a simple scaling equation for $\tilde{V}=V / \omega_{c}$ ( $\omega_{c}$ is the cutoff) follows:

$$
\frac{\partial \tilde{V}}{\partial l}=-\left(1-\frac{1}{g}\right) \tilde{V}
$$

where $l=-\log \left(\omega_{c}\right)$. Thus $\tilde{V}$ is an irrelevant operator for attractive interactions and weak barriers, when the problem scales towards a free fixed point, and the model is solvable in the low-energy limit. In addition, the weakbarrier, attractive case can be mapped onto the strongbarrier, repulsive case, and vice versa. The low energy properties of the junction are determined by the scaling of $\lim _{\omega_{c} \rightarrow 0} \tilde{V} \propto \omega_{c}^{1-1 / g}$ near the fixed point, leading to a conductance for the strong barrier repulsive case $(g<1)$ : 


$$
G \sim \omega^{\frac{2}{g}-2} \sim T^{\frac{2}{g}-2}
$$

Consider next the weak-barrier, repulsive case. The fact that the value of $g$ is not renormalized suggests a simple interpolation [5,6, 6 , between the strong-barrier and weak-barrier regimes. As $\tilde{V}$ for repulsive interactions and weak barriers grows upon scaling, we expect to eventual crossover to the case of strong barriers, so the low-energy properties of the system are described by a scaling such as eq. 2,equations like eqs. (3). As analyzed in detail below, however, this conjecture overlooks excitations that can modify the dependence shown in eqs. (3).

Since the periodic potential becomes relevant for the repulsive case, we need to infer the nature of the fixed point the Hamiltonian flows to. We assume that this fixed point is well described by the self-consistent harmonic approximation (SCHA) to this model [10], where the trial Hamiltonian reads

$\mathcal{H}_{\mathrm{SCHA}}=\frac{1}{2} \int d x\left(g \Pi(x)^{2}+g^{-1}\left(\partial_{x} \phi(x)\right)^{2}\right)+\frac{1}{2} 4 \sqrt{\pi} V^{\prime} \phi(0)^{2}$

The strength of this potential is then given by

$$
V^{\prime}=V\left(\frac{V}{\omega_{c}}\right)^{\frac{g}{1-g}}
$$

There are various reasons for this approximation being essentially correct:

- We know from the RG analysis that the system flows towards a localized regime. Any scheme that reproduces this effect, and the associated zero-point fluctuations, should be a good approximation.

- The strength of the harmonic potential $V^{\prime}$ coincides with the effective value of $V$ at the scale when $\tilde{V} \sim 1$. At this scale the flow equation (2) ceases to be valid. Thus the approximation contains, basically, the same information as the RG approach.

- If the crossover from the weak coupling to the strong coupling regimes is described by a single energy scale, this scale should be given, approximately, by eq. (5). This assumption correctly describes the low-energy dynamics of the related model of a two level system interacting ohmically with a dissipative bath [11]. The low-energy behavior of the Kondo model can also be expressed in this way 12].

In calculating the conductivity of the model, it is illuminating to analyze the new contribution to the Hamiltonian in terms of fermion operators. Using the relation for the fermion density $\rho(x)=\partial_{x} \phi / \sqrt{\pi}$, we can write

$$
\phi(0)^{2}=\pi\left(Q_{R}-Q_{L}\right)^{2} / e^{2},
$$

where $Q_{L, R}$ are the total charges on the right and left of the junction. This new term represents a chargecharge interaction, studied extensively in connection with the problem of Coulomb blockade 13, 14. To understand the origin of this term, we should note that the initial backward scattering term can be expressed as $\sim V \exp \left(2 \pi i\left(Q_{R}-Q_{L}\right) / e\right)+$ h.c.

This term suppresses small fluctuations of the charge across the junction.

For an attractive Luttinger model in the strong-barrier limit, the scaling increases the effective dimensionless hopping. Thus we expect the low-energy properties to be determined by a Hamiltonian similar to eq. (5). The low-energy modes correspond, in this case, to small fluctuations in the relative phase of the ends of the two semiinfinite systems. This situation is opposite to the one considered earlier. Upon scaling, the barriers become weaker and eventually the two chains can no longer be separated. Phase slips across the barrier are not allowed. The junction resembles a classical Josephson junction, and the harmonic potential in eq. (4) gives the plasma frequency. The bulk system, however, is gapless, because . of the dimensionality. Some of the bulk low-energy excitations hybridize with the plasma mode, modifying the properties of the junction.

For the case of weak barriers and repulsive interaction, calculation of the conductance within the framework discussed above is straightforward. The effect of an applied voltage can be described by a term in the Hamiltonian [3]

$$
\mathcal{H}_{V}=V_{\text {appl }}\left(Q_{L}-Q_{R}\right) \sim V_{\text {appl }} \phi(0),
$$

where $V_{\text {appl }}$ is the applied voltage. The conductance is given by

$$
G(\omega)=\frac{|\langle 0|j| \omega\rangle|^{2}}{\omega},
$$

where $j=e \dot{\phi}(0) / \sqrt{\pi}$,

so that $\langle 0|j| \omega\rangle \sim \omega\langle 0|\phi(0)| \omega\rangle$. The latter quantity can be obtained from the decomposition of $\phi(0)$ in normal modes. The square of the amplitude of a mode of frequency $\omega$ at the position of the barrier is proportional to the transmission coefficient of the barrier, $\tilde{T}$. A simple calculation gives

$$
\tilde{T} \sim \frac{\omega^{2}}{V^{\prime 2}},
$$

which means that the barrier is perfectly reflecting at zero energy, and that the conductance scales as

$$
G(\omega) \sim \tilde{T} \sim \omega^{2}
$$

The appearance of the $\omega^{2}$ law has been extensively discussed in connection with junctions that exhibit Coulomb blockade [14]. It is due to the small amplitude charge fluctuations across the junction. Alternatively, $\mathcal{H}_{S C H A}$ describes the damped quantum oscillator. We can use the standard analysis of this problem [15], we can write: 


$$
G(\omega)=\frac{e^{2} g}{2 \pi} \frac{\omega^{2}}{2 \pi V^{\prime 2}+g^{-2} \omega^{2}}
$$

These results can be extended to finite temperatures. A straightforward calculation (for $t \gg \hbar V^{\prime-1}$ ) gives:

$$
\begin{aligned}
2\langle\dot{\phi}(t) \dot{\phi}(0)\rangle & =\langle\{\dot{\phi}(t), \dot{\phi}(0)\}\rangle+\langle[\dot{\phi}(t), \dot{\phi}(0)]\rangle \\
& \propto \int_{0}^{\infty} d \omega \frac{\omega^{3}}{1-e^{-\beta \omega}}\left(e^{i \omega t}+e^{-\beta \omega} e^{-i \omega t}\right) \\
& =\frac{2 \pi^{4} T^{4}\left[1+2 \cosh ^{2}(\pi t T)\right]}{\sinh ^{4}(\pi t T)}+i \delta^{\prime \prime \prime}(t)
\end{aligned}
$$

where the commutator and anticommutator in this expression are related to the noise and dissipation in thermal equilibrium [16]. As the hamiltonian is harmonic, the imaginary part is independent of temperature.

The previous analysis includes only the smallamplitude charge oscillations near the minima of the potential. We can also include discrete charge transfer processes by analyzing transitions between neighboring minima (see figure 1). The wavefunction corresponding to a minimum centered around $\phi=\sqrt{\pi} n,|2 \pi n\rangle$, is given by the ground state of the hamiltonian:

$$
\mathcal{H}=\mathcal{H}_{\mathrm{SCHA}}-2 \pi n V^{\prime} \phi(0) .
$$

The hopping amplitude between neighboring minima can be calculated using the wavefunctions obtained from eq. (13) as a variational basis for the original hamiltonian, eq. (1). A correction to the ground state energy of the form $2 t_{\text {eff }} \cos (q)$ is obtained, where $q$ is the band index, and:

$$
t_{\text {eff }}=\langle 0|\mathcal{H}| 2 \pi\rangle-\langle 0|\mathcal{H}| 0\rangle\langle 0 \mid 2 \pi\rangle=V^{\prime}\langle 0 \mid 2 \pi\rangle\left(1-\frac{\pi^{2}}{4}\right)
$$

$\mathcal{H}$ is given in eq. (1). The value of the overlap, $\langle 0 \mid 2 \pi\rangle$, can be computed by noting that eqs. (4) and (13) are related by a canonical transformation. At low energies, and decomposing $\phi(0)$ in the normal modes of $\mathcal{H}_{S C H A}$ (eq. 4 ), the required transformation is:

$$
U b_{k}^{+} U^{-1}=b_{k}^{+}+\pi \sqrt{\frac{2}{g k}} .
$$

Note that $U$ does not depend on $V^{\prime}$.

From this analysis, we can infer the contribution of the interminima hopping to the junction conductivity:

$$
\hat{j}^{\prime} \propto i e V^{\prime}\left(U-U^{-1}\right)
$$

and:

$$
\left\langle\hat{j}^{\prime}(t) \hat{j}^{\prime}(0)\right\rangle \sim\left(V^{\prime} t\right)^{2 / g}
$$

This result implies that there is a contribution to the conductance which scales as $G^{\prime} \sim \omega^{2 / g-2}$. Extending the result to finite temperatures, we find a conductance which scales as: $G^{\prime} \sim T^{2 / g-2}$. Thus, the inclusion of interminima processes leads us to the expressions in the conductance derived in [3]. Alternatively, we can also say that this scaling behavior arises when the periodicity of the variable $\phi$ is restored. Note that, in terms of the backscattering potential $V$ (eq. 1), the correlation function (16) depends only on the combination $V^{1 /(1-g)} t$. This confirms the existence of a universal function which interpolates from large to small scales [3].

We can also study higher order processes induced by the residual interminima couplings. An expansion in terms of the hopping is equivalent to the expressions obtained in the strongly localized, tight binding formulation of the problem. An elegant scheme developed for the treatment of noise in this limit can be found in [17]. The study reported in [17 can also be used to compute the conductance to any order of the interminima hopping amplitude. To second order, we obtain the $G \propto \omega^{2 / g-2}$ behavior, already discussed ( and calculated for the first time in [3]). It is interesting to note that, to fourth order, keeping a finite cut-off we obtain another contribution of the type $G \propto \omega^{2}$.

This contribution is of "interdipole" origin. At finite temperatures, scaling arguments imply that the same processes lead to a $G \propto T^{2}$ dependence. The diagrams which lead to this effect were already discussed in connexion with Coulomb blockade [13,14], in the same tight binding limit.

Note that the $G \propto \omega^{2}$ dependence in the SCHA approximation does not give rise to a finite conductance at finite temperature, due to the confinement of $\phi$ induced by the parabolic potential. The interdipole processes which appear when the periodicity of $\phi$ is restored, on the other hand, do translate into a finite conductance at finite temperatures. In both cases, however, we are dealing with low amplitude charge fluctuations, in which the total charge transferred is less than the charge of one electron.

While it seems obvious that low amplitude polarization fluctuations contribute to the frequency dependence of the conductance, their role in the d. c. conductance at finite temperatures is, at first sight, somewhat unphysical. Their influence on the d. c. current requires a detailed analysis of the junction plus the external circuit. Usually, the current flowing through the junction is computed from matrix elements like $\left\langle Q\left|\mathcal{H}_{\text {tunnel }}\right| Q+e\right\rangle$ [18]. This scheme implicitly assumes that the external part of the circuit, the battery, can only gain or lose charge in discrete units. This needs not be the case. The external battery keeps the temperature, and the chemical potential fixed. Hence, its typical response times are $\hbar \tau_{\text {batt }}^{-1} \sim \max (T, e V)$. In that time, the charge which moves across the junction is $G \tau_{b a t t} V$. Thus, if $G \ll \frac{e^{2}}{\hbar} \max \left(1, \frac{T}{e V}\right)$, the battery should be able to take 
charge in arbitrary units [19].

It is illustrative to describe the two contributions to the conductivity in statistical mechanics terms. The nonanalytic term, $\omega^{2 / g-2}$, is due to topological excitations, while the analytic part, $\omega^{2}$, comes from low amplitude spin waves [20]. The latter dominates for $g<1 / 2$.

In conclusion, we present here an analysis of the strong coupling fixed point to which the tunneling hamiltonian, eq. (1), flows at low energies. The behavior of the system is shown to be closely related to that found in junctions which exhibit Coulomb blockade. We have discussed in detail the possible contributions of low energy charge fluctuations to the frequency and temperature dependence of the conductance. These processes, which involve small charge transfers across the junction, can be observed in a d. c. experiment at finite temperatures, provided that the charge at the junction cannot be considered quantized.

We are thankful to U. Weiss, for introducing us to this problem, and for many interesting discussions. This work has been supported, in part, by CICyT, Spain (grant MAT91-0905).

[1] F. P. Mulliken, C. P. Umbach and R. A. Webb, as reported in Physics Today 47, 19 (1994).

[2] F. D. M. Haldane, J. Phys. C 14, 2585 (1981).

[3] C. L. Kane and M. P. A. Fisher, Phys. Rev. Lett. 681220 (1992). Phys. Rev. B 46, 15233 (1992).

[4] A. Schmid, Phys. Rev. Lett. 51, 1506 (1983).

[5] S. A. Bulgadaev, Pis'ma Zh. Eksp. Teor. Fiz. 38, 264 (1984). English translation: Sov. Phys. JETP Lett. 39, 314 (1984).

[6] F. Guinea, V. Hakim and A. Muramatsu, Phys. Rev. Lett. 54, 263 (1985).

[7] C.H. Mak and R. Egger, Phys. Rev. E 49, 1997 (1994).

[8] "Low temperature transport of correlated electrons", R. Egger, M. Sassetti and U. Weiss, preprint.

[9] K. Moon, H. Yi, C. L. Kane, S. M. Girvin and M. P. A. Fisher, Phys. Rev. Lett. 71, 4381 (1993).

[10] M. P. A. Fisher and W. Zwerger, Phys. Rev. B 32, 6190 (1985).

[11] F. Guinea, Phys. Rev. B 32, 4486 (1985).

[12] P. Nozières, J. Low Temp. Phys. 17, 31 (1974).

[13] F. Guinea and G. Schön, Europhys. Lett. 1, 585 (1986).

[14] M. Ueda and F. Guinea, Z. Phys. B 85, 413 (1991) (note that in equation (5) of this reference, the exponent $2+g$ should be replaced by 2). F. Guinea and M. Ueda, Phys. Rev. B 49, 5722 (1994).

[15] U. Weiss, Quantum Dissipative Systems, World Scientific, Singapore (1993).

[16] C. L. Kane and M. P. A. Fisher, Phys. Rev. Lett. 72, 724 (1994).

[17] C. de C. Chamon, D. E. Freed and X. G. Wen, "Tun- neling and Quantum Noise in 1-D Luttinger Liquids ", preprint ( cond-mat/9408064).

[18] D. V. Averin and K. K. Likharev, J. Low Temp. Phys. 62, 345 (1986).

[19] Another realization is a junction which exhibits Coulomb blockade and where a voltmeter, attached to the junction measures a voltage below the charging energy ( see, for instance, L. J. Geerlings, V. F. Anderegg, C. A. Van der Jengd, J. Roomijn and J. E. Mooij, Europhys. Lett. 10, 79 (1989)). Such a measurement is only possible if neither the charge at the junction nor that at the voltmeter are quantized.

[20] Note that these fluctuations are missed if the conductivity is estimated directly from instanton proceses: S. E. Korshunov, Zh. Eksp. Toer. Fiz. 92, 1828 (1987). Engl. transl. Sov. Phys. JETP 65, 1025 (1987). In electronic systems, a similar scheme describes the transport properties in terms of the transmission coefficient, instead of using Kubo's formula. See D. Yue, L. I. Glazman and K. A. Matveev, Phys. Rev. B 49, 1966 (1994). The available Bethe ansatz approaches to the problem seem also to include only kink processes, leaving out possible contributions from low amplitude fluctuations. See P. Fendley, A. W. W. Ludwig and H. Saleur, preprint (cond-mat/9408068) and A. M. Tsvelik, preprint condmat/9409027).

Figure 1. Sketch of the parabolic potential defined in the self consistent harmonic aproximation. The arrow indicates the interminima processes which need to be considered separately (see text). 


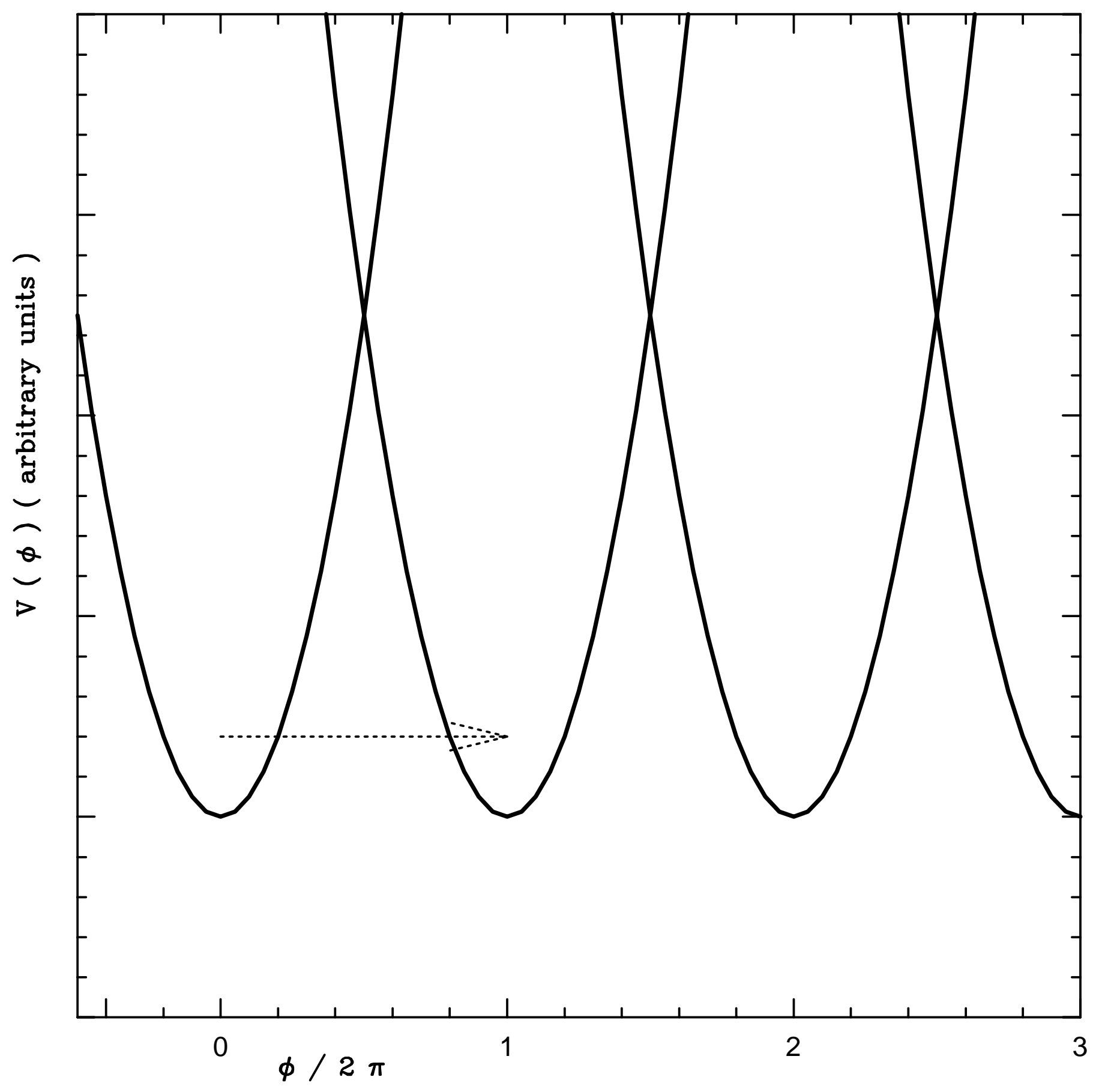

